\title{
Integrating research into clinical practice: challenges and solutions for Canada
}

\author{
François Lamontagne MD MSc, Kathryn M. Rowan PhD, Gordon Guyatt MD OC
}

Cite as: CMAJ 2021 January 25;193:E127-31. doi: 10.1503/cmaj.202397; early-released December 9, 2020

D espite Canada's investment of hundreds of millions of dollars into researching coronavirus disease 2019 (COVID-19), contributions from other countries have greatly exceeded Canada's research productivity. In contrast, the United Kingdom's National Institute for Health Research (NIHR), with its Clinical Research Network infrastructure, has engendered global envy by enabling rapid research production and knowledge dissemination within months of the start of the pandemic, which prompted calls for Canada to emulate the UK's system. ${ }^{1}$ Additional research funds in Canada have been leveraged during the pandemic, and more may be needed. However, it will take more than just funding to fulfill Canada's health research potential; a culture change is required, along with the will to forge a partnership among the provincial and territorial health systems and the various research institutes and organizations.

We discuss the limitations of the existing clinical research infrastructure in Canada, describe the mechanisms implemented to successfully embed clinical research in the UK health system and while identifying the traps that Canadian stakeholders must avoid - provide a roadmap to a Canadian version of the UK system.

\section{What impedes clinical research in Canada?}

Three main problems bedevil research processes in Canada and, if these problems remain unaddressed, future attempts to rejuvenate Canada's health research infrastructure will likely fail.

\section{Research infrastructure is inefficient}

Conduct of clinical studies (i.e., trials or prospective observational studies) require research infrastructure support. When this infrastructure is not already in place, researchers must create it at each participating site before a study can commence, which is a substantial undertaking. Planning and implementing the local research infrastructure for most clinical studies involves many stakeholders (e.g., from emergency departments, critical care departments, key consultants, pharmacies, blood banks, administrators, unions). At best, creating this infrastructure for each new clinical study is inefficient and costly. At worst, when resources, experience, or expertise are insufficient to create the infrastructure at a particular candidate clinical centre, it deprives Canadian citizens of valuable opportunities to participate in important health research, prevents Canadian scientists from developing expertise,

\section{KEY POINTS}

- Although hundreds of millions of dollars have been invested in coronavirus disease 2019 (COVID-19) research nationally, contributions from other countries have greatly exceeded Canada's research productivity.

- The UK National Institute for Health Research, with its Clinical Research Network infrastructure, has enabled rapid research production and knowledge dissemination and could be a model for Canada to emulate.

- Problems that impede the efficiency and productivity of clinical research in Canada include inefficient research infrastructure, fragmented research and a culture of research being separate from clinical practice.

- Federal and provincial political will is required to facilitate change in the culture of clinical research in Canada and to forge a partnership among the health systems and research institutes and organizations.

- A careful analysis of costs and inefficiencies in the present system, as well as thoughtful projections of potential return on investment of funding for a more efficient infrastructure, and jurisdictions willing to collaborate to try a new model, are needed to start the ball rolling.

and blocks Canadian health systems from contributing valuable evidence to guide decisions at the individual patient and health system level. Leaders at the Canadian Institutes of Health Research (CIHR) may not be fully aware of these recurrent opportunity costs and the extent to which health research funds are lost to unsuccessful projects, owing to failed attempts to create local infrastructure needed to conduct the research.

\section{Research is viewed as a separate culture}

Currently, once a clinical study begins, most health workers (e.g., nurses, physicians, allied health professionals, support staff, administrators) are engaged in it only peripherally, or not at all. Responsibility for the research falls, almost exclusively, to a small number of dedicated individuals working in a fragile ecosystem.

It is common for health workers to consider research staff as outsiders and distinct from the "genuine health system workforce." Meanwhile, those health system workers not engaged in research have limited influence on the research agenda, which contributes to a widening chasm between research and health care. 
A common perception that research is a costly diversion from clinical care exacerbates this problem and makes it difficult to persuade sites to participate in clinical studies. Furthermore, those that do participate may make limited contributions (e.g., the number of patients enrolled is small or recruitment is slow, often requiring funding extensions), and protocol adherence may be poor. ${ }^{2}$

However, because optimal effectiveness of a publicly funded health system hinges on continuous, careful evaluation of the clinical and cost effectiveness of health interventions, bridging the chasm between clinical research and health care is crucial. The COVID-19 pandemic has highlighted what happens when trustworthy evidence does not guide health decisions. At best, systems waste sparse resources; at worst, patients suffer and die. Unless health systems have a strong stake in research, other interest groups will drive the research agenda.

\section{Research efforts are fragmented}

The media have described Canada's recent experience with COVID-19 research as cacophonic or chaotic. ${ }^{3}$ Failure to coordinate research efforts invariably hinders progress, even if everything else goes well. For example, there is no mechanism in place to prevent $\mathrm{ClHR}$ committees from funding projects that compete with one another. Moreover, CIHR is not the only funder of clinical research in Canada. Whether it is the large envelopes delivered by CIHR or smaller grants awarded by not-for-profit organizations, hospital departments and research centres, funders continue to launch independent initiatives that address similar questions without consulting each other, which, in the context of limited resources, is troubling. This funding overlap and research duplication reflects a lost opportunity to federate the Canadian scientific community with respect to common objectives and to examine knowledge gaps that, without strategic planning, will remain unaddressed by ongoing studies.

\section{What's an example of an excellent national research system?}

A recent $C M A J$ editorial underscored the impressive accomplishment of the UK Randomised Evaluation of COVID-19 Therapy (RECOVERY) trial investigators and their enormous and rapid contribution to new knowledge regarding the effects of interventions for COVID-19. ${ }^{1}$ The RECOVERY trial was undertaken within the context of the UK's NIHR Clinical Research Network infrastructure. The NIHR model, which takes advantage of a preexisting, longitudinally funded clinical research network funded by and embedded within the National Health Service (NHS), simultaneously solved problems related to infrastructure development, health system engagement and fragmentation in the UK context. This infrastructure's ability to be nimble and to pivot with the onset of the pandemic allowed other ongoing trials, such as the Randomised, Embedded, Multi-factorial, Adaptive Platform trial for Community-Acquired Pneumonia (REMAPCAP), to activate more sites in the UK than in all other participating countries combined (UK sites recruited $73 \%$ of all patients with COVID-19 in the world who contributed data to REMAP-
CAP). ${ }^{4}$ Moreover, $94 \%$ of the data that informed the recent World Health Organization guidelines on corticosteroids for COVID-19 came from the UK. ${ }^{5}$

Given inherent differences between the UK and Canada, the NIHR model would require adaptation for the Canadian context. However, to inform such an adaptation, it is useful to understand the steps that led to the creation of the NIHR and appreciate the key ingredients of its success. The NIHR's scope is broad, encompassing early translational research, clinical trials and applied health and care research across multiple programs. We focus, however, on the specific components of the NIHR model designed to facilitate clinical studies such as those described above.

\section{A system born of political will}

In 2006, the UK government, aware that clinical research was performing below expectations, and after years of profound reflection regarding the role of research in health systems, ${ }^{6}$ adopted a policy whereby the health system (NHS) would become one of the main stakeholders in health research endeavours. ${ }^{7}$ To this end, where health and health research funding previously existed in silos, NHS funds were channelled to support the infrastructure required to run clinical studies across the country's health centres, including to fund structural changes (the description of which is beyond the scope of this article but discussed in depth elsewhere $\left.{ }^{8,9}\right)$. Importantly, it was the political class, rather than the scientific community, that willed these changes.

\section{Smarter infrastructure funding leading to better engagement}

In essence, the NHS repurposed funds that were "locked" in health organizations, tagged to their scientific mission (i.e., they were not used according to needs but were instead locked "into historical allocations"7), but inefficient. Instead, incremental funding supported the creation and maintenance of a clinical research network consisting of staff, facilities, equipment and support services dedicated explicitly to the delivery of clinical studies in many locations. Even if the total amount of funding had remained constant, avoiding the duplication associated with having to re-create the required infrastructure for every local clinical study would have made for considerable improved efficiency.

The result was that the health system now had an investment in clinical studies. In any system, the payer has a considerably greater influence over relevant stakeholders than a fragmented scientific community does. In effect, the NHS started leveraging this influence to increase the likelihood that every component of the system would do its share to ensure evidence-informed decision-making. Once research performance was incorporated, alongside other criteria used to allocate public funds, perceptions changed: clinicians and administrators rapidly developed a taste for good research, which substantially changed the culture of research. ${ }^{8}$

The changes also largely improved fragmented research efforts. By having to establish clear rules and processes to guide access to the new, collectively owned research infrastructure, those responsible were mindful of the need to maximize research 
efficiency. They achieved the goal by creating a portfolio of studies prioritized by representative committees and categorized by clinical areas. The NIHR closely monitors progress of prioritized studies in any given area and, until they are completed, does not offer competing projects access to the clinical research infrastructure, thus avoiding fragmentation and unproductive competition between funded studies. In addition, the NIHR has implemented processes to prevent unsuccessful studies from impeding the entire system and to encourage healthy competition among research groups. Because research funds may be terminated when set objectives are not met, researchers who are offered access to the valuable collective Clinical Research Network infrastructure must deliver results.

\section{Additional side benefits: a diversified research agenda, meritocracy and savings}

Previously, individual research groups often leveraged the track record of "their" competing research infrastructures to win funding, which perpetuated inefficient funding patterns. The new model forced established scientists to share the "ownership" of the clinical research infrastructure and broke this inefficient cycle and also made it easier for decision-makers, policy-makers, patients and members of the public to influence the research agenda. The NIHR now fosters research projects that are important for the UK population, yet still encourages scientists to offer novel ideas. Thus, the NIHR Clinical Research Network Coordinating Centre commissions (promotes) certain ideas, as well as invites (receives) ideas from researchers. This combination facilitates the incorporation of less popular research that would otherwise be unlikely to be funded, sometimes referred to as "market failures." 10

With an efficient, collectively owned research infrastructure in place, research proposals may be judged on the importance of the research question and the design of the study rather than on whether investigators have the political clout to rally a sufficient number of participating sites to complete the study. Moreover, if relevant stakeholders believe it is important to train future generations of scientists and engage health organizations, managers, clinicians, patients and the public, they can harness the potential of the national infrastructure to achieve these goals. ${ }^{11}$

Since 2006, rigorous evaluation of cost-benefit has consistently shown that the NIHR represents good value for money. Independent evaluations of the UK model concluded that "over a period from 2016/17 to $2018 / 19$, an estimated total of $£ 8$ billion in gross value added and 47467 full-time jobs were generated by Clinical Research Network-supported clinical research activity." The same analysis further highlighted that the infrastructure triggered crucial investments by the private sector, which, in turn, led to reducing system costs. ${ }^{12}$ Other studies showed that the economic benefits associated with improved health outcomes are consequential. As a result, the UK government has renewed NIHR's budget annually.

The authors of a study by the RAND Corporation concluded that "if $12 \%$ of the potential net benefit of implementing the findings of a sample of 10 studies for one year was realized, it would have covered the cost of the Programme from 1993 to
2012." 13 This argues in favour of robust economic evaluations of clinical research networks and considerations of the various ways in which better evidence may lead to cost saving. ${ }^{14}$

\section{How can a Canadian clinical research network be built?}

\section{Step 1: Initiate a dialogue}

Adapting the NIHR model to the Canadian context will require political will, as it did in the UK. In this regard, the pandemic may have planted a seed that will facilitate the start of a necessary conversation. Discussion regarding the possibility of implementing structural changes to health research in Canada should involve both federal and provincial governments. Fortunately, the basis for such discussions exists in the form of the yearly federal-provincial negotiations of funding transfers. In addition, the National Alliance of Provincial Health Research Organizations members and funders meet regularly, as do federal, provincial and territorial health ministries. A collectively owned clinical research infrastructure would provide the federal government with an important opportunity to contribute to improving Canadian health systems by attaching a portion of federal transfers to creating such a network. Provinces may consider the intersection of health and research as a welcome neutral ground. Considering that research has historically been a federal prerogative, provinces would be able to use the infrastructure to focus on some of their priorities, and better research leads to a better, more efficient and sustainable health system.

\section{Step 2: Perform baseline evaluations and benchmarking}

A comprehensive analysis of the sources of clinical research funding in Canada (e.g., CIHR, provincial health funding, private foundations) could serve as a starting point for granular discussions focused on research funding modalities. Although the absolute costs of a shared network would be considerable, so is the total amount of research funding used inefficiently in multiple, uncoordinated research projects across the country, and a fair evaluation of costs must include an estimation of the cost of the status quo. In parallel, it will be equally important to adequately map out clinical studies that currently coexist nationwide, including how long it takes to complete these studies, where patients are being recruited and the extent to which concurrent projects compete for the same research participants.

\section{Step 3: Evaluate the return on the dollar}

Applying research evidence will sometimes lead to non- or deadoption of costly interventions and choosing less expensive interventions associated with similar clinical benefits. As discussed in the UK example, greater efficiency is shown to save money. From this perspective, provinces - as stewards of the health systems in Canada - have much to gain from a network investment.

Planning how to monitor and independently evaluate research performance in Canada will therefore be crucial. Critics will note that the superiority of the NIHR model is not as convincing when there is no global health crisis but, to be fair, the ideal measure of 
national research performance is elusive. Process indicators may also be important. For example, if de-adoption of ineffective interventions, per-capita involvement of citizens participating in clinical studies, time-to-study completion, and cost of completed studies all point in the same (positive) direction, this would likely justify maintaining the new infrastructure.

\section{Step 4: Small steps, avoiding traps}

A Canada-wide consensus on emulating the UK achievement is likely to take time and may proceed piecemeal. Initially, the system could undergo pilot testing in a few willing provinces; if the experiment proves successful, others will follow. Before the pandemic, multiple strategic clinical networks were developed in Alberta; however, while this system has promoted research in principle, funding for research infrastructure was limited. After the start of the pandemic, the Quebec government created an interdisciplinary network (Réseau Québécois COVID-Pandémie) tasked with examining ways to better coordinate research efforts across multiple disciplines and sectors that have not historically been well integrated. One of the working groups is initiating "townhall meetings" focused specifically on clinical research networks. ${ }^{15}$ It is therefore possible that certain provinces, understanding that early adopters would likely exert a greater influence on the final product, would contribute proactively to creating a network. The initial Saskatchewan experiment with public insurance for physician services, which established Tommy Douglas' reputation as the father of Canadian medicare, comes to mind.

Alternatively, a pilot project could be implemented across all provinces and territories, but limited to a specific clinical area. In the United States, the National Heart, Lung, and Blood Institute's Collaborating Network of Networks for Evaluating COVID-19 and Therapeutic Strategies (CONNECTS) exemplifies this model. Although it lacks the active ingredient of research integration within a publicly funded health care system, it funds central coordination infrastructure and priority setting. It also leverages the cohesion of existing networks that may lead to important economies of scale. ${ }^{16}$ Its creation likely inspired CIHR to launch its own Canadian Network of Networks. Unfortunately, CONNECTS still has little to show for the very large sums of money invested in COVID-19 research in the US. If a Canadian Network of Networks is to prove more efficient than the American model, it must be better integrated within provincial health systems. In other words, if a Canadian Network of Networks chooses to avoid initiating discussions with both levels of government, it must ensure that resources are reserved for participating sites rather than distributing everything to researchers.

The current business model, whereby research resources are considered the private property of accomplished researchers, invariably results in pre-emptive negotiations among influential researchers who, after coming to an agreement, promote their own studies. In turn, this success, funded by the public, is integrated into subsequent funding applications as proof of their ability to build the research infrastructure required for their next project. Implicitly, this approach speaks of a broken system in which the key to academic success consists of privilege, political connections and money to sway participating sites. Although a successful track record will always be important, a perennial clinical research infrastructure will allow peer-review committees to attribute more value to the research question and the study design.

\section{Conclusion}

At the root of the currently inadequate Canadian infrastructure for research is the common view that health research and health per se are separate entities that should each exist in their own silos.

A discussion that seeks to address the scientific deficit that prevails within Canadian health systems, and its consequences, is long overdue. Better health care depends on evidenceinformed decision-making and, therefore, on high-quality clinical research responsive to the systems' needs. Our very limited success in contributing to the worldwide effort to find effective treatments for COVID-19, and discredit useless and harmful ones, have highlighted a broken system. The contrasting success of the UK model has provided us with a compelling solution to the problem through government-funded, collectively owned clinical research networks. Now is the time to act on this insight.

\section{References}

1. Murthy S, Fowler RA, Laupacis A. How Canada can better embed randomized trials into clinical care: CMAJ 2020;192:E928-E29.

2. Kasenda B, Von Elm E, You J, et al. Prevalence, characteristics, and publication of discontinued randomized trials. JAMA 2014;311:1045-51.

3. Johnson CY. Chaotic search for coronavirus treatments undermines efforts, experts say. The Washington Post 2020 Apr. 15.

4. A randomised, embedded, multi-factorial, adaptive platform trial for communityacquired pneumonia (REMAP-CAP). ClinicalTrials.gov: NCT02735707; 2016. Available: www.icnarc.org/Our-Research/Studies/Remap-Cap/About (accessed 2020 Dec. 2).

5. Lamontagne $\mathrm{F}$, Agoritsas $\mathrm{T}$, Macdonald $\mathrm{H}$, et al. A living WHO guideline on drugs for covid-19. BMJ 2020;370:m3379.

6. Soper B, Hanney SR. Lessons from the evaluation of the UK's NHS R\&D Implementation Methods Programme. Implement Sci 2007;2:7.

7. Evans TW. Best research for best health: a new national health research strategy. Clin Med (Lond) 2006;6:435-7.

8. Davies SC, Walley T, Smye S, et al. The NIHR at 10: transforming clinical research. Clin Med (Lond) 2016;16:501-2.

9. Atkinson P, Sheard S, Walley T. 'All the stars were aligned'? The origins of England's National Institute for Health Research. Health Res Policy Syst 2019;17:95. doi: 10.1186/s12961-019-0491-5.

10. Williams H. The NIHR Health Technology Assessment Programme: research needed by the NHS. Open Access Government [UK] 2020 Apr. 7. Available: www. openaccessgovernment.org/nihr-health-technology-assessment-programme -nhs/85065/ (accessed 2020 Dec. 2).

11. Clinical Research Network. UK: National Institute for Health Research. Available: www.nihr.ac.uk/explore-nihr/support/clinical-research-network.htm (accessed 2020 Dec. 2)

12. Iacobucci G. Clinical research adds billions to UK economy, analysis shows: BMJ 2019;367:16052.

13. Guthrie S, Hafner M, Bienkowska-Gibbs T, et al. Returns on research funded under the NIHR Health Technology Assessment (HTA) Programme: economic analysis and case studies. Rand Health Q 2016;5:5.

14. Bentley C, Cressman S, van der Hoek K, et al. Conducting clinical trials - costs, impacts, and the value of clinical trials networks: a scoping review. Clin Trials 2019;16:183-93.

15. Quebec COVID - Pandemic Network (RQCP). Quebec: Fonds de recherche du Quebec - Santé; 2020. Available: https://rqcp.ca/groupes-de-travail/\#recherche -clinique (accessed 2020 Dec. 2).

16. Collaborating Network of Networks for Evaluating COVID-19 and Therapeutic Strategies (CONNECTS). Bethesda (MD): National Heart, Lung, and Blood Institute; 2020. Available: www.nhlbi.nih.gov/science/collaborating-network-networks -evaluating-covid-19-and-therapeutic-strategies-connects (accessed 2020 Dec. 2). 
Competing interests: François Lamontagne has received peer-reviewed funds from the Canadian Institutes of Health Research, the Fonds de Recherche du Québec - Santé, the National Institute for Health Research (NIHR), and the Lotte \& John Hecht Memorial Foundation. Dr. Lamontagne co-led the 65 Trial funded by the NIHR Health Technology Assessment Programme (PMID 32049269) and coordinated by the Intensive Care National Audit \& Research Centre (ICNARC) Clinical Trials Unit. He is the co-scientific director of the CIHR-funded Canadian Sepsis Network, and co-director of the Réseau Québécois COVID-19 et Pandémies, funded by the Fonds de recherche du Québec Santé. Kathryn Rowan is the director/clinical trials unit director at ICNARC and honourary professor at the London School of Hygiene \&
Tropical Medicine. Dr. Rowan reports receiving research grants from NIHR Research Funding Programmes. No other competing interests were declared.

This article has been peer reviewed.

Affiliations: Université de Sherbrooke (Lamontagne); Centre de recherche du CHU de Sherbrooke (Lamontagne), Sherbrooke, Que.; Intensive Care National Audit and Research Centre (Rowan, Guyatt), London, UK; Department of Health Research Methods, Evidence and Impact (Guyatt), McMaster University Hamilton, Ont.

Contributors: François Lamontagne contributed to the conception and design of the work and drafted the manuscript. All of the authors revised it critically for important intellectual content, gave final approval of the version to be published and agreed to be accountable for all aspects of the work.

Content licence: This is an Open Access article distributed in accordance with the terms of the Creative Commons Attribution (CC BY-NC-ND 4.0) licence, which permits use, distribution and reproduction in any medium, provided that the original publication is properly cited, the use is noncommercial (i.e., research or educational use), and no modifications or adaptations are made. See: https://creativecommons. org/licenses/by-nc-nd/4.0/

Correspondence to: François Lamontagne, francois.lamontagne@usherbrooke.ca 